\title{
Servlet and Jsp
}

\author{
Yuexiu Gu \\ Qingdao University of Science and Technology, Qingdao, Shandong Province 266061, China \\ 958148232@qq.com
}

Keywords: Servlet; WEB; Jsp; WEB Container; Java

\begin{abstract}
Jsp and Servlet are often used in the WEB development, based on SUN's Java syntax rules, on the basis of most of the time in accordance with the requirements in the face of developing interface directly invoke methods, for the underlying principle and realization process of the Servlet and Jsp not to delve into. In order to more clear thorough understanding of both the clear division of labor in the WEB Container and the work process, we analysis the implementation process of the Servlet and Jsp, make application to the Servlet and Jsp development projects more handy.
\end{abstract}

\section{Introduction}

Servlet is the rules for the server side formulated by Sun Company, a java program operating on WEB Container and the universal rule that establishes connection between java program and WEB Container. [1] The full name of JSP is Java Servet Pages, and based on the server page realizing java language, the essence of JSP is Servlet. It is a standard text file and a set of rules making server translate the jsp file into java source code according to such rule, and the function is the demonstration of the completed page. [2]

\section{Features of Servlet and JSP}

Servlet. Servlet and Jsp rules are specified in order to achieve decoupling and the purpose, through this common rules so that the developer and the realization of the complete separation of the open, improve efficiency.[3]The Servlet is an interface, interface is established in the 5 methods, the most important one is service (ServletRequest req, ServletResponse res), and in the development of writing the most is this method, because it involves the preparation of logic algorithm, the other four methods in most cases is not used, so in specification in order to write code and elegant appearance, the adapter GenericServlet abstract class, in the service (ServletRequest req, ServletResponse, res) method for abstract methods, other four methods as a method of common format, and some other methods for the subclass,[4] so when writing the server at the end of the Java program, this adapter can inherit the GenericServlet abstract class, only Service (ServletRequest req,ServletResponse res override the res method, when the adapter method cannot meet the needs of the time, you can rewrite the code to make the writing more elegant. In order to ensure consistent treatment in the development of front-end transmitting data transfer request and background program, because the development of the development and development in front of the rear end is separated, the need for a rule between the two, so the background of the program attribute can get method from the front to request protocol in the http protocol for value HttpServlet Abstract class.[5]

HttpServlet extends the GenericServlet adapter and the realization of the abstract class, Serializable, Servlet, ServletConfig three interface, and in the HttpServlet abstract class defines its own unique access to the method property valuegetMethod(), but the developer is not known do not have to write code before allowing the server to send what request, also don't know the front end will send what request,so in the specification in the application of the template design pattern, HttpServlet is the most typical features in service (HttpServletRequest req, HttpServletResponse res) is established in the $\operatorname{doGet}()$ and $\operatorname{doPost}()$ call time and method, and this method of service $\operatorname{doGet}()$ and doPost() method in the method body of the exception of front and back, so it will be the core 
algorithm framework defined in advance, and specific implementation steps. The sub class is late to finish, and the algorithm is protected and reused, which fullymeets the needs of the development. ${ }^{[6]}$

Jsp. In the development of WEB, HTML code can be written in Servlet in response to the browser, but it can do so will make the HTML code and Java source program bundled with a high degree of coupling, over time in response to the page code there is a new demand, change the HTML code must recompile java source file this violates the OCP principle of opening and closing, development should be open for extension, is strictly closed for modification, hence the Jsp rules to solve the coupling problem of too high. ${ }^{[7]}$

In the development of JSP, the completed JSP file will be translated by Jsp translation engine in WEB Container into java source file and then compiled into class file. In the source file, it can be seen the source file corresponds to the class HttpJspBase class, HttpJspBase class and WEB Container implementation class is inherited from the HttpServlet abstract class, so that Jsp is the Servlet, just to work with Servlet Jsp for the different Jsp built in nine, three instructions, provides full support for grammar translation engine.

Because the translation engine does not need to shut down the server or the re deployment of the project, modify the Jsp file, JSP file translation engine will be based on the last modified time to retrieve, when accessing the JSP files on a file, the last modified time will be recorded, the last modification time the second visit will get this file, once the modification time of two times, the translation engine will start to work, JSP files will be re translated to Java source files and compiled. Class files, so HTML code written in the JSP file completely solves the coupling problem of too high, Servlet java source file is do not need to re compile in needs change. ${ }^{[8]}$

\section{Work Process under WEB Container}

After WEB Container launched, it will automatically read the web.xml file, servlet will be placed with the request path to a Map<String path, String complete class name> set, the first visit to this servlet object, reference will request path and servlet objects placed in another Map $<$ String path, Servlet servlet> set, when the user in the browser address bar in the request path, WEB Container intercepts the request path to Map<String path, Servlet servlet> collection in accordance with the request for the path to the corresponding servlet reference, if found to directly call Service() to provide services; if in the Map<String path, Servlet servlet> not found the servlet in collection, then WEB Container to Map<String path, String complete class name> find the full class name, request path corresponding to the String complete class, through the reflection mechanism, call the Servlet reference method to construct complete instantiation of the Servlet object of the ET class, and then call the object init () method to complete the initialization operation, and the information stored in the Map<String path, Servlet servlet> set, and then call service () method and logic operation service. ${ }^{[9]}$

Because Jsp Servlet essentially just does different jobs, when the server receives a request to access the JSP file, this file to get the last modified time compared with the last modification time, if the two time is different, the translation engine will JSP file into a new source file, and then compiled into Class files no reference, construction method after the translation of this servlet object will call, and then call in it () method and service () method; if the time is not the same, retranslation and compilation work, directly to find the path corresponding to the servlet object, directly call service() method, the retrieval mechanism of more than the last modification time a, also on the work folder is the active file and Class file retrieval etc.. ${ }^{[10]}$

\section{Optimization of Servlet and Jsp}

Prohibit Serlet's Automatic Loading Function. Each time you modify the Servlet, you will have to restart the server. Due to the reduced development time of the automatic loading function, the function is considered to be very useful in the development phase. However, it is very expensive in the running stage, servlet is not necessary to load, increase the burden of the class loader and cause very poor performance. Similarly, this will cause your application to be unable to collaborate with 
the class loader that has been loaded by a certain type of loader and the current class loader cannot cooperate with each other. Therefore, in order to get better performance in the running environment, the automatic loading function of the servlet is closed.

Control Http Session. Many applications require a series of client requests, so they can be related to each other. Since the HTTP protocol is stateless, Web based applications need to be responsible for maintaining such a state called session. In order to support the application of the state to be maintained, the Javaservlet technology provides the management of session and allows a variety of mechanisms to implement API's session. Http Session objects play a session, but the cost of using it requires.

Do not Use Single Thread Model. Single Thread Model makes Servlet one request at a time. If a servlet implements this interface, the servlet engine will create a separate servlet instance for the new request, which will generate a large amount of system overhead and cause stress on the server. If you need to solve the thread safety problem, use the other alternative to this interface.

\section{Conclusion}

Sevlet is Java technology's answer to CGI programming. Servlet program operates at the server end and dynamically generates Web page. Compared with traditional CGI and many similar CGI technologies, Servet is more efficient and easily to be used with more powerful functions. JSP does not add any functions that cannot essentially realized by Servlet. Besides, with the content and appearance of the decoupling, so that the front end of the page design engineers to set aside for the developer to set aside for Servlet dynamic content of the space.

\section{References:}

[1] Hu Shumin. Servlet and JSP core programming [M]. Beijing: Tsinghua University Press, 2009.

[2] Hao Yulong, J2EE programming technology [M]. Beijing: Tsinghua University Press, 2006.

[3] Lu Han, Wang Chunbin. 1200 Examples of Developing Web [M]. Beijing: Tsinghua University Press, 2011.

[4] Sun Xin. In-depth Explanation of Servlet/JSP [M]. Beijing: Electronic Industry Press, 2008.

[5] Liu Jinghua, Java Web Integrated Development [M]. Beijing: Tsinghua University Press, 2010.

[6] Susamma Barua, Teachers Assistant: A Web-based Application using Java Sever Pages and Java Database Connectivity, 2000.

[7] Henry F.K,Silberschatz A, Database System Concepts, 3nd Edition, McGrawHill, 1997.

[8] Waltz, D.L,"An English Language Questioin Answering System for a Large Relational Database", Communication of th ACM, 21.

[9] The Apache Software Foundation. The Number One HTTP Server an the Internet .http: //httpd. apache. org/.

[10]LIU Bu-xing. JSP dynamic design of garbage characters in Chinese solution [J]. Power Vocational and Technical Jour-nals, 2009. 